\title{
The effect of paired associative stimulation on fatigue resistance
}

\author{
Susanne Kumpulainen ${ }^{\mathrm{a}, *}$, Jussi Peltonen ${ }^{\mathrm{a}}$, Markus Gruber ${ }^{\mathrm{b}}$, Andrew Cresswell ${ }^{\mathrm{c}}$, \\ Sinikka Peurala ${ }^{\mathrm{d}}$, Vesa Linnamo ${ }^{\mathrm{a}}$, Janne Avela ${ }^{\mathrm{a}}$ \\ a Neuromuscular Research Center, Department of Biology of Physical Activity, University of Jyväskylä, Finland \\ b Sensorimotor Performance Lab, Department of Sport Science, University of Konstanz, Germany \\ c School of Human Movement Studies, University of Queensland, Australia \\ ${ }^{\mathrm{d}}$ Validia Rehabilitation, Lahti, Finland
}

Keywords:

Fatigue

Central fatigue

Motor cortex

Transcranial magnetic stimulation

Paired associative stimulation

\begin{abstract}
A B S T R A C T
Paired associative stimulation (PAS) is a non-invasive stimulation method developed to induce bidirectional changes in the excitability of the cortical projections to the target muscles. However, very few studies have shown an association between changes in motor evoked potentials (MEP) after PAS and behavioral changes in healthy subjects. In the present study we hypothesized that the functional relevance of PAS can be seen during fatiguing exercise, since there is always a central contribution to the development of fatigue. Transcranial magnetic stimulation was applied over the motor cortex to measure changes in the MEPs of the soleus muscle before and after PAS. Furthermore, fatigue resistance was tested during $15 \mathrm{~s}$ sustained maximal isometric contractions before and after PAS. On average, fatigue resistance did not change after PAS, however the change in excitability correlated significantly with the change in fatigue resistance. Discussion: Functionality of PAS intervention was not demonstrated in this study. However, the observed relationship between excitability and fatigue resistance suggests that PAS might have affected central fatigue during short maximal contractions.
\end{abstract}

\section{Introduction}

Paired associative stimulation (PAS) is a non-invasive method developed to induce bidirectional changes in the excitability of the cortical projections to the target muscles. PAS combines electrical stimulation of a peripheral somatosensory nerve with transcranial magnetic stimulation (TMS) over the contralateral motor cortex. Depending on the interstimulus interval (ISI), PAS can produce either long-term potentiation (LTP) - or long-term depression (LTD) - like plasticity in the target synapse (Kumpulainen et al., 2012; Stefan et al., 2000; Wolters et al., 2003), showing properties such as rapid onset, associativity, duration, specificity, and NMDA-receptor dependence (Ziemann et al., 2008). Thus, spike-timing dependent plasticity is considered the most likely mechanism behind PAS (Stefan et al., 2000; Wolters et al., 2003). PAS and motor training have been shown to share common neural mechanisms, which suggests that PAS can be used as a test for functionally relevant neuronal circuits within the motor cortex (Jung and Ziemann, 2009; Stefan et al., 2006;

* Corresponding author at: Department of Biology of Physical Activity, Box 35 40014 University of Jyväskylä, Finland. Tel.: +358 503049059; fax: +358 142602071

E-mail address: susanne.kumpulainen@jyu.fi (S. Kumpulainen).
Ziemann et al., 2004). However, very few studies have shown functionality of PAS-induced excitability changes in healthy subjects (Frantseva et al., 2008; Jung and Ziemann, 2009; Rajji et al., 2011).

Functional relevance of PAS can probably be seen during fatiguing exercises since it is well known that both central and peripheral factors contribute to the development of fatigue (Gandevia, 2001). Fatigue can be defined as any exercise-induced reduction in the ability of a muscle to generate maximal force or power (Gandevia, 2001). Central fatigue refers to processes proximal to the neuromuscular junction and peripheral fatigue to processes at or distal to it (Gandevia, 2001). The relative contribution of the central and peripheral components depends on the intensity and duration of the fatiguing exercise. Short maximal sustained contractions have been shown to have a substantial central contribution to the development of fatigue (Gandevia et al., 1996; Hunter et al., 2006, 2008; Lentz and Nielsen, 2002; Szubski et al., 2007; Taylor et al., 1996, 1999). Central fatigue has been defined as a progressive reduction in the voluntary activation of a muscle during exercise and it can originate at both spinal and supraspinal levels (Gandevia, 2001). Previous studies suggest that central fatigue at least partially originates from inadequate cortical drive to the motor neurons (Gandevia, 2001; Hunter et al., 2006, 2008). 


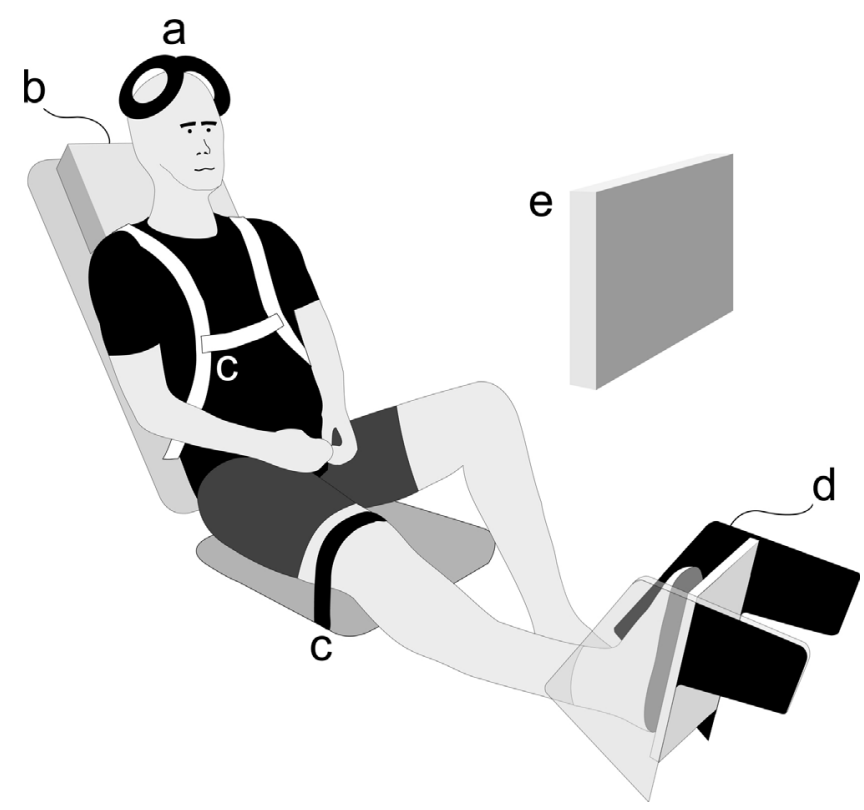

Fig. 1. Schematic of the measurement setup in the ankle dynamometer. (a) A stimulating coil was placed and secured over the left hemisphere and (b) subject's neck was comfortably supported by a head rest. (c) Body movement was restricted with seat belts and a knee strap. (d) Right foot was installed to a force pedal and (e) the force was displayed on a computer screen in front of the subject.

Because PAS can be used to systematically alter the responsiveness of neurons in the primary motor cortex, the current study was designed to investigate the effect of two different PAS interventions on fatigue resistance during $15 \mathrm{~s}$ sustained maximal isometric contractions. The PAS interventions targeted the soleus muscle (SOL) as this is an important antigravity muscle during standing and a major contributor to force production during the impact phase of walking and running (Ishikawa et al., 2005). It was hypothesized

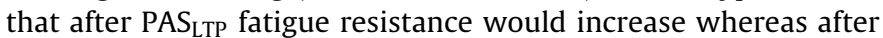
PAS $_{\text {LTD }}$ fatigue resistance would decrease.

\section{Materials and methods}

\subsection{Ethical approval and subjects}

Thirty healthy subjects volunteered for the study and were divided into two groups: PAS induced LTP-like plasticity group $\left(\mathrm{PAS}_{\mathrm{LTP}} ; 9\right.$ females and 6 males, $25 \pm 4$ years, $62 \pm 10 \mathrm{~kg}$, $168 \pm 11 \mathrm{~cm})$ and LTD-like plasticity group $\left(\mathrm{PAS}_{\mathrm{LTD}} ; 9\right.$ females and 6 males, $25 \pm 4$ years, $63 \pm 7 \mathrm{~kg}, 168 \pm 7 \mathrm{~cm}$ ). Subjects were blinded to the PAS intervention they were undergoing and thus the PAS $\mathrm{STT}_{\mathrm{LT}}$ and PAS $_{\text {LTD }}$ were considered as each other's control. None of the subjects had any history of neuromuscular or orthopedic diseases and all subjects were naïve to the experiments. Before testing, subjects were informed about the procedures and gave written consent. The study was approved by the ethics board from the University of Jyväskylä and was performed in conformity with the latest revision of the declaration of Helsinki.

\subsection{Experimental design}

Participants were positioned on a custom built ankle dynamometer (University of Jyväskylä, Finland) with the hip at $110^{\circ}$, the knee in an extended position at $180^{\circ}$, the ankle at $90^{\circ}$ and the right foot resting on a pedal (Fig. 1). A seat belt restricted movement of the upper body and straps secured the right leg and foot. Hands were resting in the lap during all measurements. Prior to the measurements, the participants performed three maximal isometric plantar flexions with a 3 min rest interval between trials. The highest force value was considered as the maximal voluntary contraction (MVC). The experimental protocol is shown in Fig. 2; the upper panel represents the main protocol and the lower panel the additional procedures for sub-groups. The main protocol included transcranial magnetic stimulation (TMS) to measure changes in the motor evoked potentials (MEPs) of SOL before (pre) and twice after the PAS intervention; immediately after (post0) and $15 \mathrm{~min}$ after (post15) PAS. The fatigue resistance tests were performed before (pre) and after all TMS measurements (post15). To test for changes at the spinal level, SOL Hoffman reflexes (H-reflexes) were elicited in a subgroup at the pre, post 0 and post15 measurements. Fatigue, MEP and PAS procedures were conducted so that fatigue would not affect MEPs or PAS intervention. To avoid possible fatigue effects there was at least $40 \mathrm{~min}$ between the last fatiguing contraction and the PAS intervention in the pre measurements. In the post measurements, fatigue resistance was measured after all the MEPs were recorded but within $25 \mathrm{~min}$ of the PAS protocol, because LTP/LTD effects have been shown to last for a minimum of $30 \mathrm{~min}$ (Kumpulainen et al., 2012; Mrachacz-Kersting et al., 2007; Stefan et al., 2000; Wolters et al., 2003).

\subsection{Recordings}

For electromyographic (EMG) measurements, a pseudomonopolar electrode placement protocol was used where one surface electrode of a pair (Unilect, $\mathrm{Ag} / \mathrm{AgCl}$, Unomedical Ltd., Redditch, UK) was placed on the right SOL and the other over a bony surface of the tibia. A ground electrode was placed over the lateral malleolus (Hoffman et al., 2009). The pseudomonopolar setup allowed MEPs of higher amplitude to be recorded, which in turn also decreased the intensity of the stimulus needed to evoke a detectable MEP. Prior to electrode placement, the skin was shaved, abraded and cleaned with alcohol to reduce resistance below $5 \mathrm{k} \Omega$. EMG signals were amplified $(100 \times)$, band-pass filtered $(10-1000 \mathrm{~Hz})$ and sampled at $5 \mathrm{kHz}$ (Neural Systems NL 900D and NL 844, Digitimer Ltd., Hertfordshire, UK). EMG data and reaction forces from the pedal were collected with a computer via 16-bit AD converter (CED power 1401, Cambridge Electronics Design Limited, UK) and stored for later analysis.

\subsection{Procedures}

A rectangular current pulse with a duration of $1 \mathrm{~ms}$ was delivered to the common tibial nerve using a constant-current stimulator (DS7AH, Digitimer Ltd., Hertfordshire, UK) for the PAS protocol in addition to evoking $\mathrm{H}$-reflexes and maximal M-waves $\left(M_{\max }\right)$. A circular cathode with a pickup area of $77 \mathrm{~mm}^{2}$ (Unilect short-term ECG Electrodes, $\mathrm{Ag} / \mathrm{AgCl}$, Unomedical Ltd., UK) was placed over the tibial nerve on the popliteal fossa and an oval shaped $(5.08 \mathrm{~cm} / 10.16 \mathrm{~cm}$ ) anode ( $\mathrm{V}$-trodes neurostimulation electrodes, Mattler Electronics Corp., USA) was placed above the patella. Motor threshold (MT) was defined as the minimal intensity that induced a visually identifiable muscle twitch in SOL. To quantify reliable $M_{\max }$, supramaximal stimulus intensity was used, being $150 \%$ of the current needed to elicit maximal stimulus response.

For the fatigue test subjects were instructed to produce their maximal isometric plantar flexion force and maintain it for $15 \mathrm{~s}$ (Fig. 3), during which the force declined toward the end. Verbal encouragement was given throughout the trial. At the end of the fatiguing contraction, neural deficit was estimated using the interpolation twitch technique (ITT); a supramaximal ( $M_{\max }$ intensity) double pulse with $10 \mathrm{~ms}$ interval was delivered to the tibial nerve to quantify possible increment in force (superimposed twitch). 


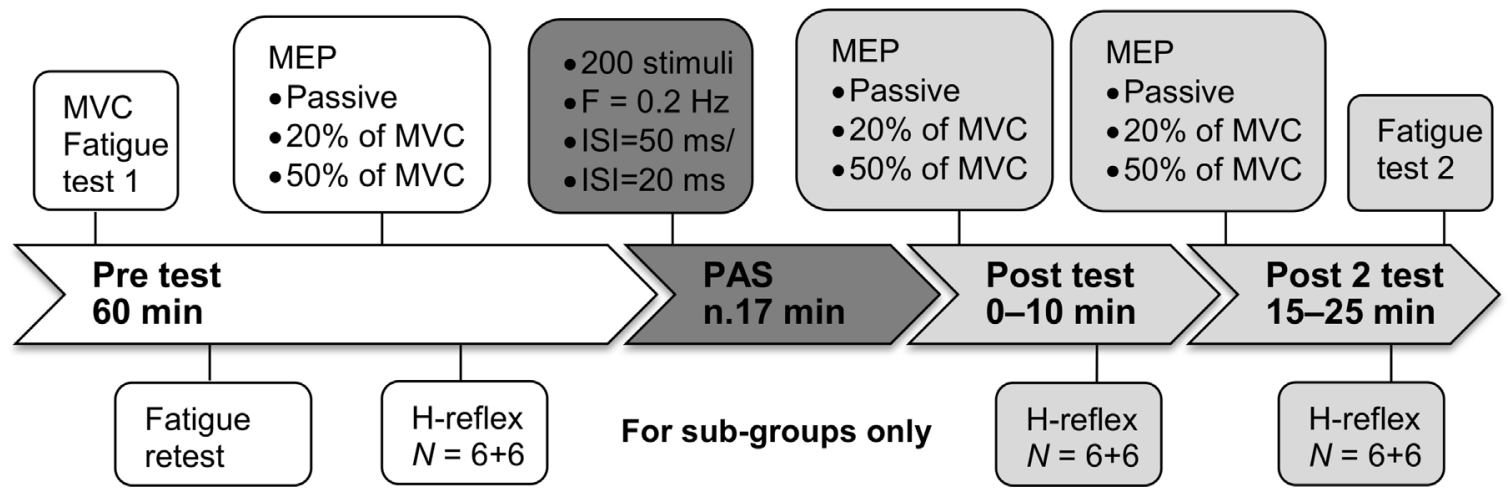

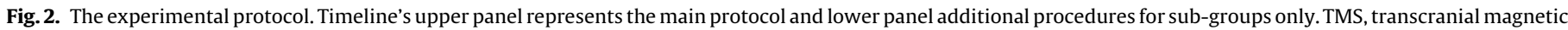
stimulation; MVC, maximal voluntary contraction; ISI, inter-stimulus interval; PAS, paired associated stimulation.

Immediately following the sustained MVC, the same supramaximal double pulse was delivered to the relaxed muscle to quantify resting twitch. Neural deficit indicates insufficient central drive to the motor neurons and is attributable to central fatigue. Test-retest repeatability was determined by repeating the fatigue resistance test prior to intervention in ten subjects. There was a mandatory break of 10 min between the test and the retest to reduce the possibility of any fatigue effect. It has been shown in previous studies that 10 s sustained maximal force is recovered within $5 \mathrm{~min}$ (Benwell et al., 2006).

TMS was delivered using a mono-pulse Magstim 2002 stimulator with a $9 \mathrm{~cm}$ double batwing coil (Magstim, Whitland, UK). The optimal stimulus site for SOL was usually located $1 \mathrm{~cm}$ lateral and $1 \mathrm{~cm}$ posterior to the vertex. A custom-made coil holder and rubber straps were used to fix the coil firmly to the head. The position of the coil was marked on a closely fitted cap worn by the subjects. The cap also protected the hair and head from cold spray (PRF101, Taerosol, Kangasala, Finland), which was used to prevent the coil from overheating. Resting motor threshold (RMT) was defined as the lowest stimulus intensity required to elicit a MEP with a peakto-peak amplitude of $50 \mu \mathrm{V}$ in three out of five consecutive trials. Stimulus intensity was then set to $120 \%$ of RMT and this intensity was used throughout the experiment. TMS was delivered ten times to passive muscle and five times to active muscle at $20 \%$ of MVC and $50 \%$ of MVC. It has been previously demonstrated that MEP responses increase progressively from rest to a contraction strength of $80 \%$ of MVC in the SOL (Oya et al., 2008). As the force-time curve was displayed on the screen in front of the subjects, they were able to reach their target level with an accuracy of $\pm 2 \%$ during the active conditions.

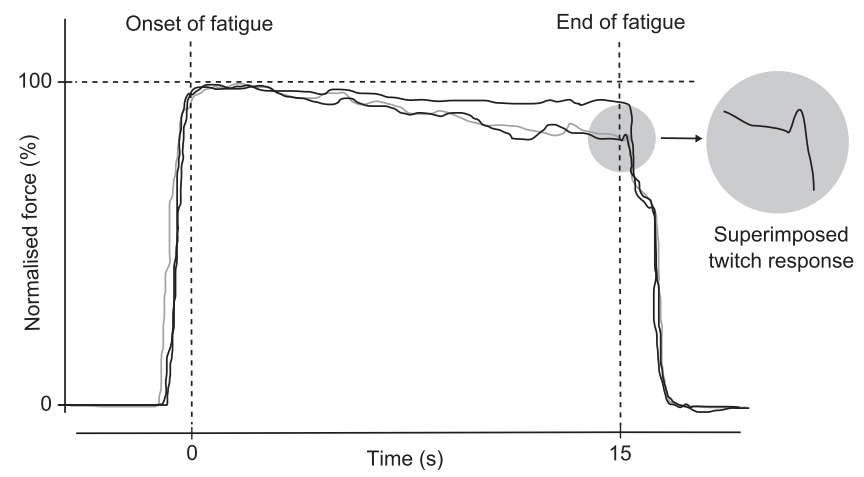

Fig. 3. Example figure of the fatigue resistance test. Force traces are from one representative subject. Two united thin lines demonstrate the test-retest repeatability prior to intervention (fatigue test 1 = black line, fatigue retest = gray line). The thicker black line represents the post-intervention test. Force $(F)$ is normalized to maximal voluntary force.
$\mathrm{H}$-reflexes were measured in sub-groups of six subjects from both PAS $_{\text {LTP }}$ and PAS $_{\text {LTD }}$ groups during rest. $M_{\max }$ was elicited before all H-reflex measurements. Then 10 submaximal stimuli were applied with a current intensity that evoked peak-to-peak M-wave responses of $20 \pm 5 \%$ of $M_{\max }$ to quantify the H-reflex.

PAS consisted of a single electrical stimulation delivered to the tibialis nerve at $150 \%$ of MT and a single TMS pulse at $120 \%$ of RMT. ISIs to induce LTP- and LTD-like plasticity were selected based on previous experiments. A constant ISI of $50 \mathrm{~ms}$ was used for the PAS $_{\text {LTP }}$ group (Kumpulainen et al., 2012; Mrachacz-Kersting et al., 2007; Poon et al., 2008) and constant ISI of $20 \mathrm{~ms}$ was used for the PAS LTD group (Jayaram and Stinear, 2008; Poon et al., 2008; Stinear and Hornby, 2005). A total of 200 pairs of stimuli were applied at a rate of $0.2 \mathrm{~Hz}$. To optimize the PAS effect, subjects were asked to produce $5 \%$ of MVC plantar flexion with their right leg during the PAS protocol (Mrachacz-Kersting et al., 2007).

\subsection{Data analysis and statistics}

Commercially available software (Spike2, CED, Cambridge, England) was used for all offline analyses. Fatigue resistance was calculated by dividing the average force during the $15 \mathrm{~s}$ isometric contraction by the individual MVC. Thus fatigue resistance of $100 \%$ corresponds to the theoretical situation where the initial MVC force is maintained for the entire $15 \mathrm{~s}$. Amplitudes of the superimposed twitch and resting twitch were measured and the ratio between them was calculated to estimate neural deficit. To determine the efficacy of PAS, peak-to-peak MEP amplitudes were measured from SOL and averaged. Spinal efficacy was determined by taking $\mathrm{H}-$ reflex and $M_{\max }$ peak-to-peak amplitudes and calculating the ratio of these two measures $\left(H / M_{\max }\right)$. Cortical silent period (SP) was analyzed when MEPs were delivered during contractions at $50 \%$ of MVC as recommended by Säisänen et al. (2008) and also during contractions at $20 \%$ of MVC. SP refers to a silencing of any ongoing EMG activity after the MEP as a result of TMS being delivered during a voluntary contraction. The duration of the SP was determined by visual inspection as the time from MEP offset to the time of reoccurrence of voluntary EMG activity. Each individual trial was analyzed separately and then averaged across trials and subjects.

Normality of variables was tested with Shapiro-Wilk's $W$ tests. Accordingly, SOL MEPs, neural deficit and $H / M_{\max }$ were compared using Wilcoxon's signed-rank test separately for both groups. Differences between groups for PAS effect (post MEPs as percentage of baseline values) were tested with Mann-Whitney $U$-tests. MVC and fatigue force were compared with two-tailed paired $t$ test. Fatigue resistance was compared with a two-way repeated measures ANOVA with within factor time of two levels 
(pre PAS/post PAS) and between-subjects factor group of two levels $\left(\mathrm{PAS}_{\mathrm{LTP}} / \mathrm{PAS}_{\mathrm{LTD}}\right)$. SPs were analyzed with a two-way repeated measures ANOVA with within factor time of three levels (pre PAS/post0 PAS/post15) and between-subjects factor group of two levels $\left(\mathrm{PAS}_{\mathrm{LTP}} / \mathrm{PAS}_{\mathrm{LTD}}\right)$. Spearman's coefficient was used to correlate the changes in PAS-induced excitability (at post15; MEPs as percentage of baseline values) and changes in fatigue resistance. The inter-class correlation coefficient (ICC) was calculated for fatigue resistance test-retest repeatability. In a secondary analysis the above-mentioned tests for neural correlates and fatigue resistance were also performed only for the PAS responders (post $\mathrm{MEP} /$ pre MEP ratio $>1.00$ in the $\mathrm{PAS}_{\mathrm{LTP}}$ group and $<1.00$ in the PAS $_{\text {LTD }}$ group). The secondary analysis was done due to the high inter-individual variability of the PAS effects, which have been previously reported (Ridding and Ziemann, 2010). The significance level was set at $P<0.05$. All data are given as mean \pm standard deviation (SD).

\section{Results}

\subsection{Change in excitability}

Fig. 4 shows original resting MEP recordings for one representative subject in the PAS ${ }_{\text {LTP }}$ and PAS ${ }_{\text {LTD }}$ groups before, immediately

A



C

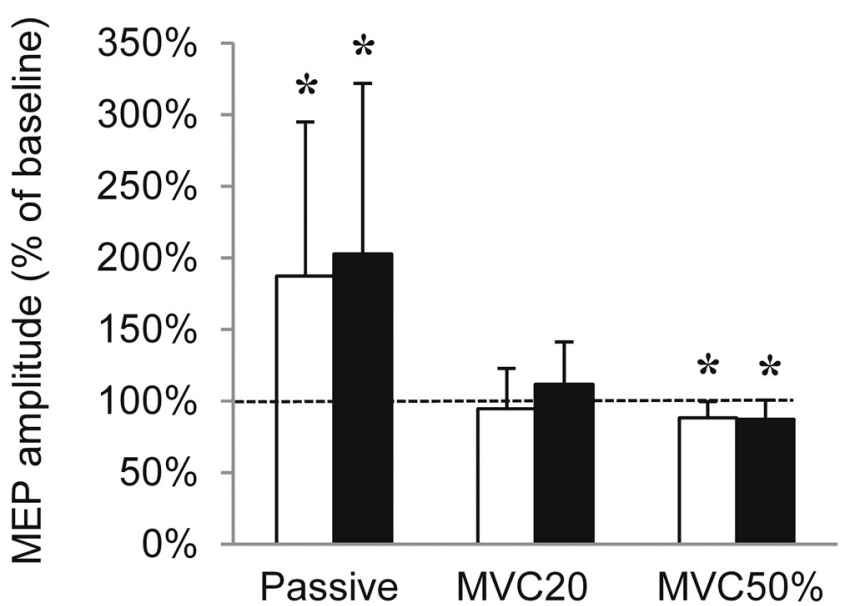

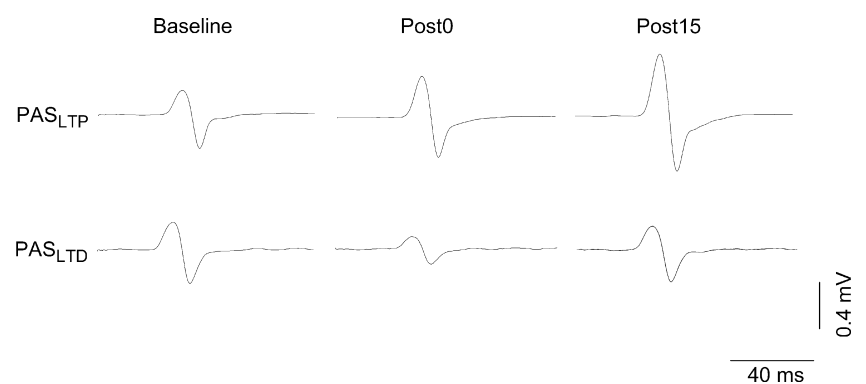

Fig. 4. Resting MEP traces. Traces are from one representative subject in the $P_{A S}$ and PAS ${ }_{\text {LTD }}$ groups before and after the interventions. Each trace is an average of 10 trials.

after and 15 min after PAS. The PAS effect (post MEPs/pre MEPs) was significantly different between groups at post 0 and post 15 at rest $(P<0.05)$ but not in active conditions $(P>0.05)$. The normalized post-intervention peak-to-peak MEP amplitudes are presented in


post0 $(P>0.05)$ and significantly by $73 \pm 123 \%(P<0.05)$ at post 15 at rest. There were no significant changes in the $20 \%$ of MVC condition but MEPs decreased significantly at both post 0 by $9 \pm 12 \%$ $(P<0.05)$ and post15 by $8 \pm 14 \%(P<0.05)$ in the $50 \%$ of MVC

B

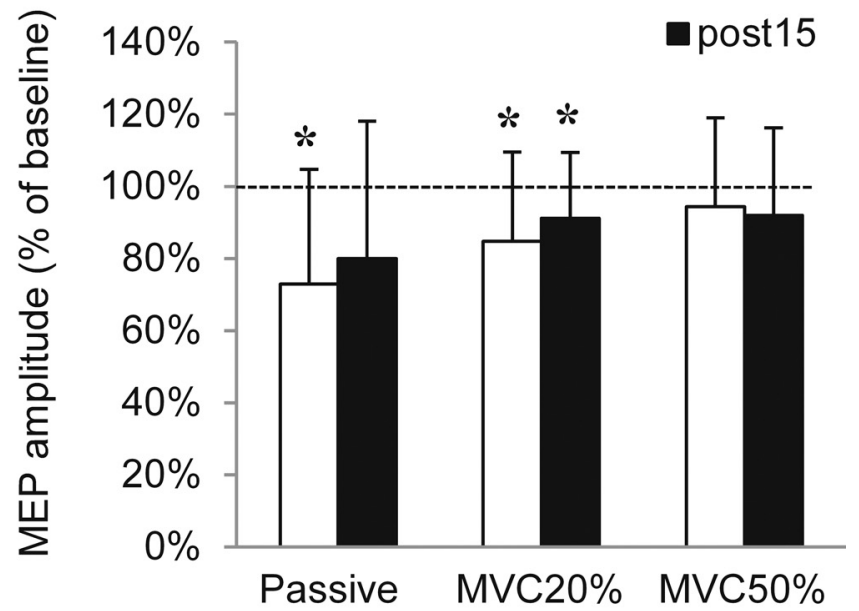

D

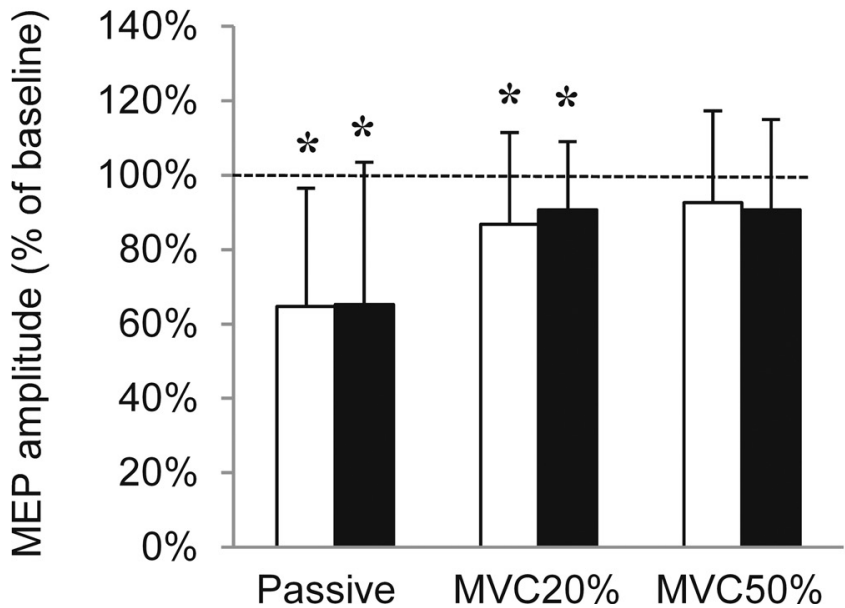

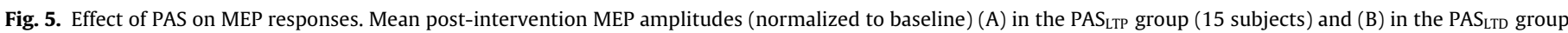

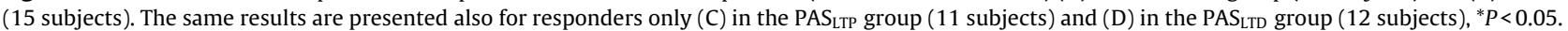




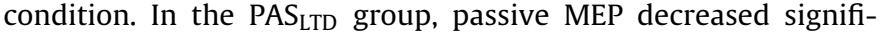
cantly at post 0 by $27 \pm 32 \%(P<0.05)$ but not at post 15 , where MEP decreased by $20 \pm 38 \%(P>0.05)$. MEP decreased significantly by $15 \pm 25 \%(P<0.05)$ at post0 and by $9 \pm 18 \%(P<0.05)$ in the $20 \%$ of MVC condition. There were no significant changes in the $50 \%$ condition. The difference between the post0 and post 15 was never statistically significant. SPs at contraction level of 50\% of MVC were not affected by time $\left(F_{(2,56)}=1.54, P>0.05\right)$ group $\left(F_{(1,28)}=0.87, P>0.05\right)$ nor their interaction $\left(F_{(2,56)}=0.06, P>0.05\right)$. SP values for the PAS $\mathrm{PTP}_{\mathrm{LT}}$ group were $75 \pm 27 \mathrm{~ms}$ at pre, $78 \pm 23 \mathrm{~ms}$ at post 0 and $79 \pm 25 \mathrm{~ms}$ at post 15 , and for the PAS ${ }_{\text {LTD }}$ group $68 \pm 19 \mathrm{~ms}, 72 \pm 15 \mathrm{~ms}$ and $73 \pm 14 \mathrm{~ms}$, respectively. Correspondingly, there were no changes in SP duration at $20 \%$ of MVC; time $\left(F_{(2,56)}=1.83, P>0.05\right)$ group $\left(F_{(1,28)}=1,66, P>0.05\right)$ nor their interaction $\left(F_{(2.56)}=0.19, P>0.05\right)$. The coefficient of variation was significantly higher, 0.25 at $20 \%$ of MVC compared to 0.16 at $50 \%$ of MVC $(P<0.05)$. Neither $M_{\max }$ amplitude nor $H / M_{\max }$ ratio changed significantly throughout the protocol $(P>0.05) . M_{\max }$ values for the PAS $\mathrm{STP}_{\text {LT }}$ group were $19 \pm 3 \mathrm{mV}$ at pre, $18 \pm 3 \mathrm{mV}$ at post0 and $18 \pm 3 \mathrm{mV}$ at post15, and for the $\mathrm{PAS}_{\mathrm{LTD}}$ group $19 \pm 3 \mathrm{mV}$, $19 \pm 3 \mathrm{mV}$ and $19 \pm 3 \mathrm{mV}$, respectively. $H / M_{\max }$ ratios for the PAS $\mathrm{LTP}_{\mathrm{L}}$ group were $0.60 \pm 0.07$ at pre, $0.58 \pm 0.12$ at post 0 and $0.60 \pm 0.09$ at post15, and for the PAS ${ }_{\text {LTD }}$ group $0.49 \pm 0.24,0.47 \pm 0.22$ and $0.51 \pm 0.24$, respectively. The secondary analysis with only responders is shown in Fig. 5C and B; MEPs increased significantly at both post0 and post15 $(P<0.05)$ in the PAS LTP $_{\text {group }(11 \text { subjects }) \text { and }}$ decreased significantly at both post 0 and post15 $(P<0.05)$ in the PAS $_{\text {LTD }}$ group (12 subjects). Otherwise the significances in MEP and SP results did not differ from the whole group analysis.

\subsection{Fatigue resistance}

In both groups the $15 \mathrm{~s}$ fatigue test induced significant force

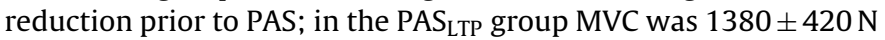
and average fatigue force was significantly less, $1230 \pm 340 \mathrm{~N}$ $(P<0.001)$. In the PAS ${ }_{\text {LTD }}$ group, MVC force was $1190 \pm 320 \mathrm{~N}$ and average fatigue force was significantly less, $1110 \pm 280 \mathrm{~N}$ $(P<0.01)$. Corresponding fatigue resistance values were $90 \pm 8 \%$

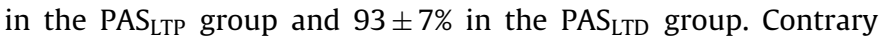
to our hypothesis, fatigue resistance was not affected by time $\left(F_{(1,28)}=0.11, P>0.05\right)$ group $\left(F_{(1,28)}=0.59, \quad P>0.05\right)$ nor their interaction $\left(F_{(1,28)}=0.86, P>0.05\right)$. After the PAS interventions, fatigue resistance was $92 \pm 1 \%$ in the PAS $\mathrm{STP}_{\mathrm{LT}}$ group and $92 \pm 7 \%$ in the PAS $\mathrm{STD}_{\text {LT }}$ group. However, when the groups were combined, the change in excitability (post15 MEPs as a percentage of baseline values) correlated significantly with the change in fatigue resistance $(N=30, R=0.40, P<0.05)$, which can be seen in Fig. 6 . In the PAS $\mathrm{LTP}_{\mathrm{L}}$ group, neural deficit was $1.6 \pm 2.4 \%$ prior to PAS and $0.9 \pm 4.4 \%$

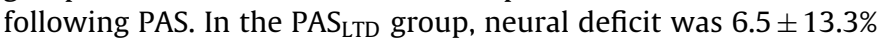
prior to PAS and $5.9 \pm 11.2 \%$ following PAS. Neural deficit decreased non-significantly $(P>0.05)$ by $44 \pm 79 \%$ in PAS $_{\text {LTP }}$ and by $10 \pm 30 \%$ in PAS $_{\text {LTD. }}$ Test-retest comparison $(N=10)$ revealed excellent repeatability of the fatigue resistance test $(P<0.001)$. When only responders $\left(\mathrm{PAS}_{\mathrm{LTP}}\right.$ group $=11$ subjects and $\mathrm{PAS}_{\mathrm{LTD}}$ group $=12$ subjects) were included in the secondary analysis, two-way repeated measures of ANOVA revealed a significant interaction of time and group $\left(F_{(1,21)}=4.8, P<0.05\right)$. Post hoc analysis using two-tailed paired $t$-test showed a non-significant improvement in fatigue resistance by $3.1 \pm 8.4 \%$ in the $\mathrm{PAS}_{\mathrm{LTP}}$ group and a reduction of $3.0 \pm 7.5 \%$ in the PAS $_{\text {LTD }}$ group. Otherwise the significances in force results did not differ from the whole group analysis.

\section{Discussion}

The aim of this study was to investigate the functional relevance of PAS by determining the effect of two different PAS intervention

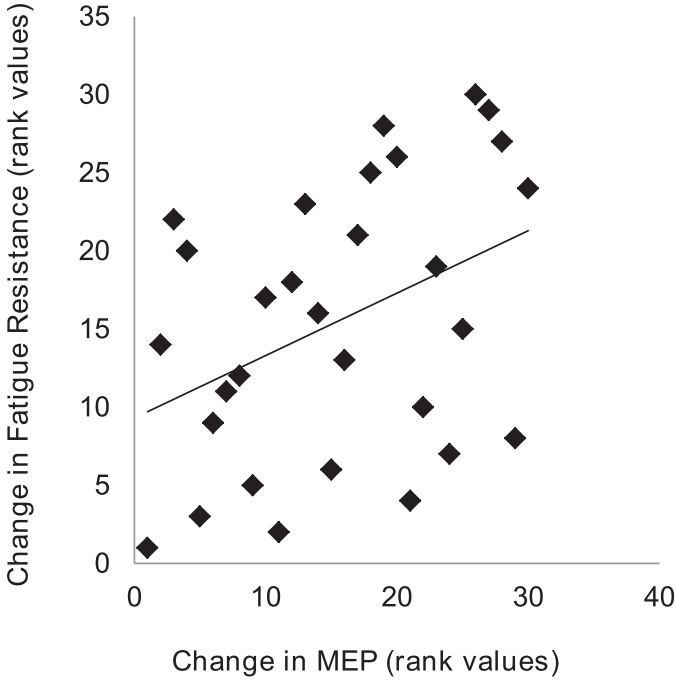

Fig. 6. Relationship between excitability and fatigue resistance. Spearman's coefficient was used to correlate PAS-induced changes in excitability, presented on the horizontal axis, with changes in fatigue resistance, presented on the vertical axis $(N=30, R=0.40, P<0.05)$.

on fatigue resistance during 15 s sustained maximal isometric contraction. On average fatigue resistance did not change after $\mathrm{PAS}_{\mathrm{LTP}}$

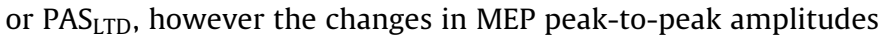
after PAS correlated with the changes in fatigue resistance. Subjects whose MEP size increased also demonstrated improved fatigue resistance and vice versa.

\subsection{Neural correlates - changes in cortico-spinal and spinal excitabilities after PAS}

The results of the present study indicate that associative modulation of excitability to the cortical projections of SOL was achieved after PAS ${ }_{\text {LTP }}$ and PAS ${ }_{\text {LTD }}$ interventions with constant ISIs.

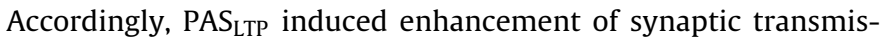

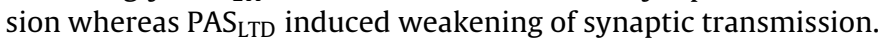
There was no significant increase in MEP size at post0 after PAS $\mathrm{LTP}_{\mathrm{LP}}$, but a significant increase at post15. A similar trend has been seen in previous studies that have shown an increasing PAS $_{\text {LTP }}$ effect over time (Kumpulainen et al., 2012; Prior and Stinear, 2006; Stefan et al., 2000). On the contrary, MEP size decreased signif-

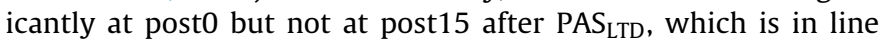
with a previous study by Di Lazzaro et al. (2011) where a significant effect was achieved immediately after but not 30 min after

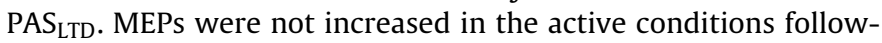

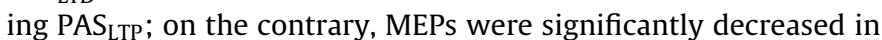

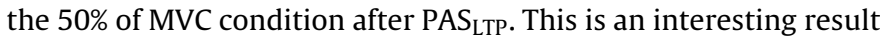
since, to our knowledge, PAS-induced effects have not been measured at such high contraction levels, and a significant decrease

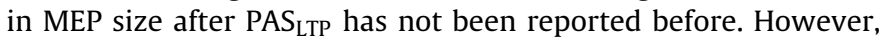
there are studies showing no change in MEPs during slight contractions (Kumpulainen et al., 2012; Stefan et al., 2000, 2004). The interpretation of the effect of PAS on active muscles is more complicated as can be seen in the study of Lu et al. (2009) where a decrease of the movement-related cortical potentials (MRCP) in electroencephalography recordings was reported after an LTPlike PAS-protocol. Accordingly, performing the same movement pattern, simple thumb abduction, generated a decreased MRCP negativity after the PAS intervention. MRCP reflects executive aspects of the forthcoming motor action and decreased MRCP negativity indicates weaker volitional motor output. This may indicate that the LTP-like effect decreases the level of effort needed to 
produce force, resulting in decreased or unchanged MEPs in active muscle.

The most stable and informative SP is measured at a contraction level of $50 \%$ of MVC (Säisänen et al., 2008). It has been shown that the initial part of the SP is influenced by spinal circuitries, whereas the latter part to a higher extent represents the state of cortical inhibitory interneurons, which are mediated by GABA ${ }^{\mathrm{b}}$ receptors (Ziemann, 2004). SP remained unchanged in the current study, which is in line with the study by Di Lazzaro et al. (2011) that reported unchanged SPs after PAS LTP $_{\text {or PAS }}$ LTD when MEPs were elicited during contraction at $50 \%$ of MVC. $H / M_{\max }$ ratios remained unchanged after the interventions, suggesting a lack of changes at the spinal level, which has also been shown in previous PAS studies (Kumpulainen et al., 2012; Stefan et al., 2000; Wolters et al., 2003).

\subsection{Functional implications - changes in fatigue resistance after} PAS

The $15 \mathrm{~s}$ sustained maximal isometric contraction induced significant force loss on all occasions but fatigue resistance did not change after interventions. This is in line with a previous study by Milanović et al. (2011) which showed no changes in a fatigue test involving sustained isometric contraction at $50 \%$ of MVC after

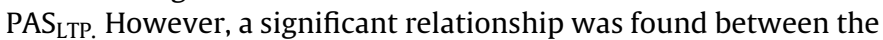
change in excitability of the cortical projections and change in fatigue resistance in the present study, which was not reported in the study by Milanović et al. (2011). One possible reason for this difference could be that they used the duration of submaximal contraction as an indication of fatigue, which may involve a greater peripheral component of fatigue compared to the present protocol (Lentz and Nielsen, 2002). It has been demonstrated that endurance time and changes in maximal capacity to generate force provide information about different processes induced by the exercise (Vøllestad, 1997). Thus, endurance time may not directly correlate with motor cortex excitability. Benwell et al. (2006) found a significant reduction in the rate of force loss during a 10-s MVC of hand muscles after increasing corticomotor excitability using a spike timing-dependent repetitive TMS intervention. In the current study the lack of changes in fatigue resistance at the group level may be due to considerable inter-individual variability in the PASinduced plasticity changes within the groups. The chosen constant ISIs might be one reason for relatively high inter-individual variability (SD $= \pm 108 \%$ and $123 \%$ ) observed in this study. Nevertheless, the present values are comparable to the variability $(S D= \pm 105 \%$ and $52 \%$ ) and number of responders (12 responders from 16 subjects) obtained in a previous study (Kumpulainen et al., 2012), where the ISI was optimized to the individual somatosensoryevoked potential and was $50 \pm 2 \mathrm{~ms}$ on average. Since an earlier study by Mrachacz-Kersting et al. (2007) found significant LTP during a $10 \mathrm{~ms}$ time window with constant ISIs of $45-55 \mathrm{~ms}$, the constant ISI of $50 \mathrm{~ms}$ used here should be sufficient to induce LTP in most subjects. The optimal ISI to induce LTD in the leg area has not been studied but an ISI of $20 \mathrm{~ms}$ is mostly used. Stinear and Hornby (2005) reported the number of LTD responders and it was quite similar to ours; 12 responders from 14 subjects. Also previous studies have reported wide variability of PAS-induced effects between subjects, which has been associated with differences in brain anatomy, genes, and training background (Ridding and Ziemann, 2010). Because of the dissimilarities in brain anatomy, TMS can preferentially activate different neuronal circuits in different subjects (Sakai et al., 1997) causing variability in the PAS-induced effects. The genetic polymorphisms of neurotrophins can influence the induction of plasticity (Ridding and Ziemann, 2010). However, there is only one brain-derived neurotrophic factor - polymorphism, which has been shown to limit PAS-induced motor cortex plasticity (Cheeran et al., 2008). Furthermore, it has been found that skill athletes have higher motor cortex plasticity compared to endurance trained athletes (Kumpulainen et al., 2014). When only responders were included in the analysis, there was significant interaction of time and group in fatigue resistance. Still, the change in fatigue resistance was not significant within the groups. This suggests that PAS affects fatigue resistance among PAS responders and supports the result of significant correlation. ITT was conducted in order to reveal possible changes in neural deficit induced by PAS $_{\text {LTP }}$ or PAS LTD $_{\text {at }}$ at the end of fatiguing contractions. No significant differences were found, suggesting that there were no differences in central fatigue after the interventions. However, since we found a significant correlation between changes in MEP and fatigue resistance, small changes in central fatigue may have occurred within the variation of ITT. Low sensitivity of the ITT method has been reported at maximal contraction intensities (Taylor, 2009).

\subsection{Candidate mechanisms behind PAS and fatigue}

It has been proposed that PAS-induced LTP occurs within the motor cortex (Di Lazzaro et al., 2009; Stefan et al., 2000), where the somatosensory stimulus can arrive via the dorsal column-medial leminiscal route or via a longer pathway involving the cerebellum (Strigaro et al., 2014). Intracortical neural circuits through which the effects of PAS and fatigue emerge have been investigated with single and paired pulse TMS in several previous studies (Carson and Kennedy, 2013; Gruet et al., 2013; Taylor and Gandevia, 2001). Paired pulse TMS is a tool to examine facilitatory and inhibitory circuits in the cerebral cortex. However, as different studies have used different interventions and target muscles, the results are difficult to interpret. Prolongation of SP has been observed after various fatiguing exercises including sustained MVC of soleus muscle (Iguchi and Shields, 2012; McKay et al., 1996). Since prolongation of SP is less after cervicomedullary stimulation-induced MEP, additional inhibition at the cortical level has been suggested (Levenez et al., 2008; Taylor et al., 1996). In addition, Hilty et al. (2011) showed that central projections of group III-IV muscle afferents may facilitate a fatigue-induced increase in SP. Therefore, it seems that $\mathrm{GABA}^{\mathrm{b}}$-mediated intracortical inhibitory circuits have a role in the development of central fatigue. Since SP duration remained unchanged after PAS interventions in this study, it seems that, at least partly, fatigue and PAS affect different cortical interneurons. This corresponds to the current results showing no significant effect of PAS on fatigue resistance. However, among PAS responders the PAS-induced LTP might have compensated for fatigue-induced inhibition in the motor cortex and thus affected fatigue resistance. In addition, PAS induced LTP/LTD-like plasticity has been shown to be accompanied with other neuronal circuits in the cerebral cortex (Carson and Kennedy, 2013), which might have contributed to the observed significant correlation. For example, long afferent inhibition (LAI), which is also GABA ${ }^{\mathrm{b}}$-mediated, is decreased fol-

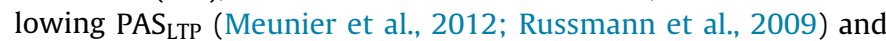
might increase motor drive to the exercising muscle during fatiguing tasks. LAI reflects the activity of somatosensory inputs, and is obtained when the interval between peripheral afferent stimulation and subsequent TMS is in the region of $200 \mathrm{~ms}$.

\section{Conclusions}

In the present study, PAS induced associative plasticity changes in the cortical projections to the resting SOL. On average, fatigue resistance during a 15-s sustained maximal isometric contraction did not change following PAS interventions. Thus, functionality of PAS interventions was not evident with the current experimental design. However, among responders fatigue resistance showed 
significant interaction of time and group and PAS-induced excitability changes correlated significantly with changes in fatigue resistance. This suggests that PAS might have slightly affected central fatigue during short maximal contractions. Therefore, PAS might have implications for improving performance in rehabilitation settings.

\section{Acknowledgments}

The authors are very grateful to the subjects who participated in the study and to the laboratory staff from the Neuromuscular Research Center (Department of Biology of Physical Activity) of the University of Jyväskylä, Finland, for their valuable contributions to this project.

\section{References}

Benwell, N.M., Mastaglia, F.L., Thickbroom, G.W., 2006. Paired-pulse rTMS at transsynaptic intervals increases corticomotor excitability and reduces the rate of force loss during a fatiguing exercise of the hand. Exp. Brain Res. 175, 626-632.

Carson, R.G., Kennedy, N.C., 2013. Modulation of human corticospinal excitability by paired associative stimulation. Front. Hum. Neurosci. 7.

Cheeran, B., Talelli, P., Mori, F., Koch, G., Suppa, A., Edwards, M., Houlden, H., Bhatia, K., Greenwood, R., Rothwell, J.C., 2008. A common polymorphism in the brainderived neurotrophic factor gene (BDNF) modulates human cortical plasticity and the response to rTMS. J. Physiol. 586, 5717-5725.

Di Lazzaro, V., Dileone, M., Pilato, F., Profice, P., Oliviero, A., Mazzone, P., Insola, A., Capone, F., Ranieri, F., Tonali, P.A., 2009. Associative motor cortex plasticity: direct evidence in humans. Cereb. Cortex 19, 2326-2330.

Di Lazzaro, V., Dileone, M., Pilato, F., Capone, F., Musumeci, G., Ranieri, F., Ricci, V., Bria, P., Di Iorio, R., de Waure, C., Pasqualetti, P., Profice, P., 2011. Modulation of motor cortex neuronal networks by rTMS: comparison of local and remote effects of six different protocols of stimulation. J. Neurophysiol. 105, 2150-2156.

Frantseva, M.V., Fitzgerald, P.B., Chen, R., Moller, B., Daigle, M., Daskalakis, Z.J., 2008. Evidence for impaired long-term potentiation in schizophrenia and its relationship to motor skill learning. Cereb. Cortex 18, 990-996.

Gandevia, S.C., 2001. Spinal and supraspinal factors in human muscle fatigue. Physiol. Rev. 81, 1725-1789.

Gandevia, S.C., Allen, G.M., Butler, J.E., Taylor, J.L., 1996. Supraspinal factors in human muscle fatigue: evidence for suboptimal output from the motor cortex. J. Physiol 490 (Pt 2), 529-536.

Gruet, M., Temesi, J., Rupp, T., Levy, P., Millet, G., Verges, S., 2013. Stimulation of the motor cortex and corticospinal tract to assess human muscle fatigue. Neuroscience 231, 384-399.

Hilty, L., Lutz, K., Maurer, K., Rodenkirch, T., Spengler, C.M., Boutellier, U., Jäncke, L., Amann, M., 2011. Spinal opioid receptor-sensitive muscle afferents contribute to the fatigue-induced increase in intracortical inhibition in healthy humans. Exp. Physiol. 96, 505-517.

Hoffman, B.W., Oya, T., Carroll, T.J., Cresswell, A.G., 2009. Increases in corticospinal responsiveness during a sustained submaximal plantar flexion. J. Appl. Physiol (1985) 107, 112-120.

Hunter, S.K., Todd, G., Butler, J.E., Gandevia, S.C., Taylor, J.L., 2008. Recovery from supraspinal fatigue is slowed in old adults after fatiguing maximal isometric contractions. J. Appl. Physiol. (1985) 105, 1199-1209.

Hunter, S.K., Butler, J.E., Todd, G., Gandevia, S.C., Taylor, J.L., 2006. Supraspinal fatigue does not explain the sex difference in muscle fatigue of maximal contractions. J. Appl. Physiol. (1985) 101, 1036-1044.

Iguchi, M., Shields, R.K., 2012. Cortical and segmental excitability during fatiguing contractions of the soleus muscle in humans. Clin. Neurophysiol. 123, 335-343.

Ishikawa, M., Komi, P.V., Grey, M.J., Lepola, V., Bruggemann, G.P., 2005. Muscletendon interaction and elastic energy usage in human walking. J. Appl. Physiol. (1985) 99, 603-608.

Jayaram, G., Stinear, J., 2008. Contralesional paired associative stimulation increases paretic lower limb motor excitability post-stroke. Exp. Brain Res. 185, 563-570.

Jung, P., Ziemann, U., 2009. Homeostatic and nonhomeostatic modulation of learning in human motor cortex. J. Neurosci. 29, 5597-5604.

Kumpulainen, S., Mrachacz-Kersting, N., Peltonen, J., Voigt, M., Avela, J., 2012. The optimal interstimulus interval and repeatability of paired associative stimulation when the soleus muscle is targeted. Exp. Brain Res. 221, 241-249.

Kumpulainen, S., Avela, J., Gruber, M., Bergmann, J., Voigt, M., Linnamo, V., MrachaczKersting, N., 2014. Differential modulation of motor cortex plasticity in skill-and endurance-trained athletes. Eur. J. Appl. Physiol., 1-9.

Lentz, M., Nielsen, J.F., 2002. Post-exercise facilitation and depression of M wave and motor evoked potentials in healthy subjects. Clin. Neurophysiol. 113, 1092-1098.
Levenez, M., Garland, S.J., Klass, M., Duchateau, J., 2008. Cortical and spinal modulation of antagonist coactivation during a submaximal fatiguing contraction in humans. J. Neurophysiol. 99, 554-563.

Lu, M., Bliem, B., Jung, P., Arai, N., Tsai, C., Ziemann, U., 2009. Modulation of preparatory volitional motor cortical activity by paired associative transcranial magnetic stimulation. Hum. Brain Mapp. 30, 3645-3656.

McKay, W.B., Stokic, D.S., Sherwood, A.M., Vrbova, G., Dimitrijevic, M.R., 1996 Effect of fatiguing maximal voluntary contraction on excitatory and inhibitory responses elicited by transcranial magnetic motor cortex stimulation. Muscle Nerve 19, 1017-1024.

Meunier, S., Russmann, H., Shamim, E., Lamy, J., Hallett, M., 2012. Plasticity of cortica inhibition in dystonia is impaired after motor learning and paired-associative stimulation. Eur. J. Neurosci. 35, 975-986.

Milanović, S., Filipović, S., Blesić, S., Ilić, T., Dhanasekaran, S., Ljubisavljević, M., 2011. Paired-associative stimulation can modulate muscle fatigue induced motor cortex excitability changes. Behav. Brain Res. 223, 30-35.

Mrachacz-Kersting, N., Fong, M., Murphy, B.A., Sinkjaer, T., 2007. Changes in excitability of the cortical projections to the human tibialis anterior after paired associative stimulation. J. Neurophysiol. 97, 1951-1958.

Oya, T., Hoffman, B.W., Cresswell, A.G., 2008. Corticospinal-evoked responses in lower limb muscles during voluntary contractions at varying strengths. J. Appl. Physiol. (1985) 105, 1527-1532.

Poon, D., Roy, F., Gorassini, M., Stein, R., 2008. Interaction of paired cortical and peripheral nerve stimulation on human motor neurons. Exp. Brain Res. 188 $13-21$.

Prior, M.M., Stinear, J.W., 2006. Phasic spike-timing-dependent plasticity of human motor cortex during walking. Brain Res. 1110, 150-158.

Rajji, T.K., Liu, S., Frantseva, M.V., Mulsant, B.H., Thoma, J., Chen, R., Fitzgerald, P.B., Daskalakis, Z.J., 2011. Exploring the effect of inducing long-term potentiation in the human motor cortex on motor learning. Brain Stimul. 4, 137-144

Ridding, M.C., Ziemann, U., 2010. Determinants of the induction of cortical plasticity by non-invasive brain stimulation in healthy subjects. J. Physiol. 588, 2291-2304.

Russmann, H., Lamy, J., Shamim, E.A., Meunier, S., Hallett, M., 2009. Associative plasticity in intracortical inhibitory circuits in human motor cortex. Clin. Neurophysiol. 120, 1204-1212.

Säisänen, L., Pirinen, E., Teitti, S., Könönen, M., Julkunen, P., Määttä, S., Karhu, J., 2008 Factors influencing cortical silent period: optimized stimulus location, intensity and muscle contraction. J. Neurosci. Methods 169, 231-238.

Sakai, K., Ugawa, Y., Terao, Y., Hanajima, R., Furubayashi, T., Kanazawa, I., 1997. Preferential activation of different I waves by transcranial magnetic stimulation with a figure-of-eight-shaped coil. Exp. Brain Res. 113, 24-32.

Stefan, K., Wycislo, M., Classen, J., 2004. Modulation of associative human motor cortical plasticity by attention. J. Neurophysiol. 92, 66-72.

Stefan, K., Wycislo, M., Gentner, R., Schramm, A., Naumann, M., Reiners, K., Classen, J., 2006. Temporary occlusion of associative motor cortical plasticity by prior dynamic motor training. Cereb. Cortex 16, 376-385.

Stefan, K., Kunesch, E., Cohen, L.G., Benecke, R., Classen, J., 2000. Induction of plasticity in the human motor cortex by paired associative stimulation. Brain 123, $572-584$

Stinear, J.W., Hornby, T.G., 2005. Stimulation-induced changes in lower limb corticomotor excitability during treadmill walking in humans. J. Physiol. 567 701-711.

Strigaro, G., Hamada, M., Murase, N., Cantello, R., Rothwell, J.C., 2014. Interaction between different interneuron networks involved in human associative plasticity. Brain Stimul. 7, 658-664.

Szubski, C., Burtscher, M., Loscher, W.N., 2007. Neuromuscular fatigue during sustained contractions performed in short-term hypoxia. Med. Sci. Sports Exerc. 39, 948-954.

Taylor, J.L., Gandevia, S.C., 2001. Transcranial magnetic stimulation and human muscle fatigue. Muscle Nerve 24, 18-29.

Taylor, J.L., Butler, J.E., Gandevia, S., 1999. Altered responses of human elbow flexors to peripheral-nerve and cortical stimulation during a sustained maximal voluntary contraction. Exp. Brain Res. 127, 108-115.

Taylor, J.L., 2009. Point: the interpolated twitch does/does not provide a valid measure of the voluntary activation of muscle. J. Appl. Physiol. (1985) 107, 354-355

Taylor, J.L., Butler, J.E., Allen, G.M., Gandevia, S.C., 1996. Changes in motor cortica excitability during human muscle fatigue. J. Physiol. 490 (Pt 2), 519-528.

Vøllestad, N.K., 1997. Measurement of human muscle fatigue. J. Neurosci. Methods 74, 219-227.

Wolters, A., Sandbrink, F., Schlottmann, A., Kunesch, E., Stefan, K., Cohen, L.G., Benecke, R., Classen, J., 2003. A temporally asymmetric Hebbian rule governing plasticity in the human motor cortex. J. Neurophysiol. 89, 2339-2345.

Ziemann, U., Ilic, TV , Pauli, C., Meintzschel, F., Ruge, D, 2004. Learning modifies subsequent induction of long-term potentiation-like and long-term depressionlike plasticity in human motor cortex. J. Neurosci. 24, 1666-1672.

Ziemann, U., 2004. TMS and drugs. Clin. Neurophysiol. 115, 1717-1729.

Ziemann, U., Paulus, W., Nitsche, M.A., Pascual-Leone, A., Byblow, W.D., Berardelli, A., Siebner, H.R., Classen, J., Cohen, L.G., Rothwell, J.C., 2008. Consensus: motor cortex plasticity protocols. Brain Stimul. 1, 164-182. 